\title{
INTEGRABILITY AND GLOBAL DYNAMICS OF THE MAY-LEONARD MODEL
}

\author{
GAMALIEL BLÉ ${ }^{1}$, VÍCTOR CASTELLANOS ${ }^{1}$, JAUME LLIBRE ${ }^{2}$ AND \\ INGRID QUILANTÁN ${ }^{1}$
}

\begin{abstract}
We study when the celebrated May-Leonard model in $\mathbb{R}^{3}$, describing the competition between three species and depending on two positive parameters $a$ and $b$, is completely integrable; i.e. when $a+b=2$ or $a=b$. For these values of the parameters we shall describe its global dynamics in the compactification of the positive octant, i.e. adding its infinity.

If $a+b=2$ and $a \neq 1$ (otherwise the dynamics is very easy) the global dynamics was partially known, and roughly speaking there are invariant topological half-cones by the flow of the system. These halfcones have vertex at the origin of coordinates and surround the bisectrix $x=y=z$, and foliate the positive octant. The orbits of each half-cone are attracted to a unique periodic orbit of the half-cone, which lives on the plane $x+y+z=1$.

If $b=a \neq 1$ then we consider two cases. First, if $0<a<1$ then the unique positive equilibrium point attracts all the orbits of the interior of the positive octant. If $a>1$ then there are three equilibria in the boundary of the positive octant, which attract almost all the orbits of the interior of the octant, we describe completely their bassins of attractions.
\end{abstract}

\section{INTRODUCTION}

The Lotka-Volterra systems are classical differential systems introduced independently by Lotka and Volterra in the 1920s to model the interaction among species, see [15, 24], see also Kolmogorov [12]. They are equivalent to the replicator differential equations used in game theoretic applications to economics and evolution. See the good book of Hofbauer and Sigmund [8] for an introduction to the Lotka-Volterra and replicator systems and their applications. Nowdays the Lotka-Volterra systems continue being intensively studied see for instance the recent works on them in $[2,9,11,16,22,23]$ where are studied from different points of view as their phase portraits, oscillations, traveling wave solutions, with delay, ...

A particular class of the 3-dimensional Lotka-Volterra systems are the so called May-Leonard models. More precisely, in 1975 May and Leonard [17]

2010 Mathematics Subject Classification. 34D45, 34D05, 37N25, 92D25, 34C12, 34C30.

Key words and phrases. May-Leonard model, Lotka-Volterra systems, first integrals, global dynamics, Poincaré compactification. 
studied the 3-dimensional Lotka-Volterra differential system

$$
\begin{aligned}
& \dot{x}=x(1-x-a y-b z), \\
& \dot{y}=y(1-b x-y-a z), \\
& \dot{z}=z(1-a x-b y-z),
\end{aligned}
$$

in $x \geq 0, y \geq 0$, and $z \geq 0$, describing the competition between three species and depending on two parameters $a>0$ and $b>0$. See [17] for the biological meaning of the variables $x, y, z$ and the parameters $a$ and $b$. Differential system (1) has been extensively studied by several authors, for instance see $[1,7,10,13,18,20]$ and the references therein.

Let $\mathbb{R}_{+}=[0, \infty)$. Assume that $a+b>2$ and either $a<1$ or $b<1$. The carrying simplex $\mathbb{S}$ is the boundary in $\mathbb{R}_{+}^{3}$ of the basin of repulsion of the origin of the differential system (1). $\mathbb{S}$ is also the boundary in $\mathbb{R}_{+}^{3}$ of the basin of repulsion of the infinity. It is an invariant 2-dimensional surface, homeomorphic to the standard unit simplex, whose boundary contained in $\{x=0\} \cup\{y=0\} \cup\{z=0\}$ attracts all positive orbits except the positive equilibrium point, for more details see $[17,6,26,21]$; this boundary was called by May and Leonard a special class of attracting periodic limit cycle solution. In fact it is an attractor heteroclinic cycle in modern language of the qualitative theory of differential equations, detected by first time in a Lotka-Volterra system becomes the May-Leonard model so celebrated. Thus, up to now, the article [17] has been quoted in 516 papers or books, see the Google Scholar.

Our objective is to study the completely integrable systems inside the May-Leonard model (1), and to describe its global dynamics in the compactification of $\mathbb{R}_{+}^{3}$ in function of the parameters $a$ and $b$. Roughly speaking the Poincaré ball is obtained identifying $\mathbb{R}^{3}$ with the interior of the 3 -dimensional ball of radius one centered at the origin of coordinates, and extending analytically the flow of the May-Leonard model to the boundary $\mathbb{S}^{2}$ of this ball, and consequently to the infinity. In this way we can study the behavior of the May-Leonard model in a neighborhood of the infinity, and describe completely the global dynamics of that differential system. For a precise information on the Poincaré compactification see the appendix.

We want to mention that the global description of the flow of a differential system in $\mathbb{R}^{3}$ is usually very difficult and generally impossible. Here, using the compactification of Poincaré and the existence of a first integral that we know explicitly, such a global description is possible for this differential system coming from a relevant biological model. The way followed for providing the global phase portrait of this system can be applied to many other systems, specially if they exhibits some first integrals.

The region of biological interest in the May-Leonard model is the first octant of $\mathbb{R}^{3}$ which its closure in the Poincaré ball is identified with

$$
R=\left\{(x, y, z) \in \mathbb{R}^{3}: x^{2}+y^{2}+z^{2} \leq 1, x \geq 0, y \geq 0 z \geq 0\right\},
$$

and we shall describe the dynamics of the May-Leonard model in $R$. 
We shall describe the global dynamics of the May-Leonard differential system in the octant $R$ for the following values of the parameters $a+b=2$ or $b=a$. In the first case and if $a \neq 1$ (otherwise the dynamics is very easy, see Proposition 3 ) the global dynamics was partially known. We complete its study describing the dynamics at infinity, and roughly speaking showing that there are invariant topological half-cones by the flow of the system. These half-cones have vertex at the origin of coordinates and surround the bisectrix $x=y=z$, and they foliate the octant $R$. The orbits of each half-cone are attracted to a unique periodic orbit of the cone, which lives on the plane $x+y+z=1$, see Theorem 2 .

If $b=a \neq 1$ then we consider two cases. First, if $0<a<1$ then the equilibrium point $(1 /(1+2 a), 1 /(1+2 a), 1 /(1+2 a))$ attracts all the orbits of the interior of the octant $R$, see Theorem 4 . If $a>1$ then there are three equilibria $(1,0,0),(0,1,0)$ and $(0,0,1)$ in the boundary of $R$, which attract almost all the orbits of $R$, we describe completely their bassins of attractions, see Theorem 4 .

The reason for which we can describe completely the global dynamics of the May-Leonard differential system for the values of the parameters satisfying $a+b=2$ or $a=b$ is that for these values the system is completely integrable, see Theorem 1.

We obtain the following new biological conclusions. First, it was known that for $a+b<2$ there was a local stable positive equilibrium in the octant $R$, we have improved this when additionally we have that $a=b$, because then the bassin of attraction of this stable positive equilibrium is the whole interior of the octant $R$, so it is a global attractor in the interior of $R$. Hence, under these values of the parameters $a=b<1$ the three species persist and tend to an equilibrium point.

Second, it was known that for $a>1$ and $b>1$ there were local stable equilibria in each one of the finite boundaries $x=0, y=0$ and $z=0$ of $R$, and consequently that there was conditions for which one specie disappears and the other two remain. Our results for $a=b>1$ show that the bassins of attraction of these three stable equilibria located in such boundaries fill the whole interior of the octant $R$ except a surface which separates the bassins. We provide the explicit expression of this surface.

\section{Statement of the main Results}

Let $U$ be an open and dense subset of $R$. We say that a $C^{1}$ function $H: U \rightarrow \mathbb{R}$ non-constant in any open subset of $U$ is a first integral of the differential system (1) if $H(x, y, z)$ is constant, for all the values of $t$ for which the solution $(x(t), y(t), z(t))$ is defined and contained in $U$. Let

$$
\mathcal{X}=P^{1}(x, y, z) \frac{\partial}{\partial x}+P^{2}(x, y, z) \frac{\partial}{\partial y}+P^{3}(x, y, z) \frac{\partial}{\partial z}
$$


be the differential operator associated to the vector field (1), then

$$
\begin{aligned}
& P^{1}(x, y, z)=x(1-x-a y-b z), \\
& P^{2}(x, y, z)=y(1-b x-y-a z), \\
& P^{3}(x, y, z)=x(1-a x-b y-z) .
\end{aligned}
$$

Clearly a $C^{1}$ function $H: U \rightarrow \mathbb{R}$ non-constant in any open subset of $U$ is a first integral of the differential system (1) if and only if $\mathcal{X} H=0$ in $U$.

Let $H_{1}: U_{1} \rightarrow \mathbb{R}$ and $H_{2}: U_{2} \rightarrow \mathbb{R}$ be two first integrals of the differential system (1), we say they are independent in $U_{1} \cap U_{2}$ if their gradients are independent in all the points of $U_{1} \cap U_{2}$ except perhaps in a zero Lebesgue measure set. The differential system (1) is completely integrable in $R$ if it has two independent first integrals. In this case the orbits of the differential system (1) are contained in the curves $\left\{H_{1}(x, y, z)=h_{1}\right\} \cap\left\{H_{2}(x, y, z)=\right.$ $\left.h_{2}\right\}$, when $h_{1}$ and $h_{2}$ vary in $\mathbb{R}$.

As far as we know the firsts in studying the first integrals of the MayLeonard model were Leach and Miritzis [13] (see also [14]), who obtained the following first integrals:

(i) $H_{1}=\frac{x y z}{(x+y+z)^{3}}$ if $a+b=2$ and $a \neq 1$,

(ii) $H_{2}=\frac{y(x-z)}{x(y-z)}$ if $a=b \neq 1$,

(iii) $H_{3}=x / z$ and $H_{4}=y / z$ two independent first integrals if $a=b=1$.

Our first result is:

Theorem 1. The May-Leonard differential system (1) in $R$ is completely integrable if either $a+b=2$, or $b=a$.

Theorem 1 is proved in section 3 .

We remark that we have analytic expressions depending on a quadrature for the second first integral provided by the Jacobi Theorem, see section 3 and section 2.11 of [5]. But these quadratures are not easy in both cases $a+b=2$ and $b=a$.

The exact knowledge of the dynamics of the May-Leonard differential system for the values of the parameters $a$ and $b$ when $a+b=2$ or $b=a$, provide many information about the dynamics of these systems when the parameters $a$ and $b$ are sufficiently near to this straight lines in the quadrant $\{(a, b): a>0, b>0\}$ of the parameters.

In order to describe the global dynamics of the May-Leonard differential system in $R$ we need to describe the $\alpha$-and $\omega$-limits of all orbits of system (1) in $R$. For a precise definition of the $\alpha$-limit and of the $\omega$-limit of an orbit, see for instance section 1.4 of [4].

Since the planes of coordinates and the infinity are invariant by the Poincaré compactification of system (1), the four boundaries of $R$ are invariant. As usual we denote the boundary of $R$ by $\partial R$. The flow on these boundaries and in the interior of $R$ is described in the next theorem when 
$a+b=2$. For a definition of topologically equivalent phase portraits see for instance section 1.3 of [4].

If $A$ is a subset of $\mathbb{R}^{3}$, its image inside the Poincaré ball $B$ through the identification of $\mathbb{R}^{3}$ with the interior of this ball is denoted by $p(A)$. Thus for instance the plane $\{x+y+z=1\}$ of $\mathbb{R}^{3}$ inside the Poincaré ball is denoted by $p(\{x+y+z=1\})$.

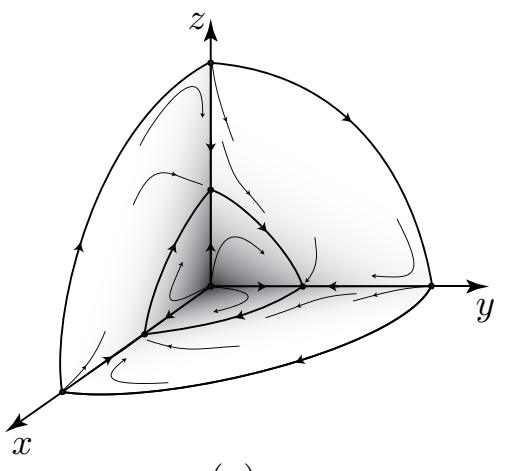

(a)

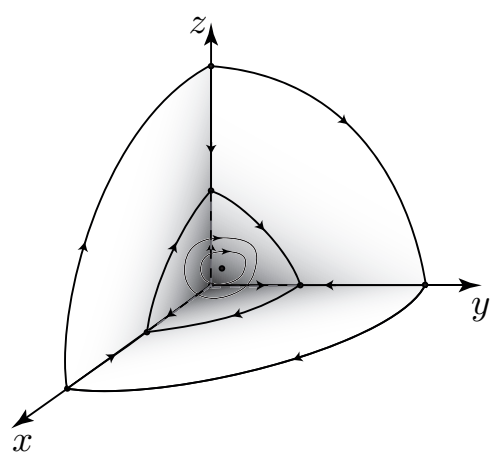

(c)

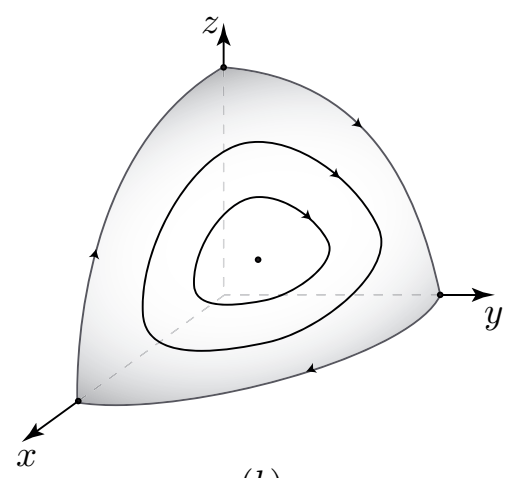

(b)

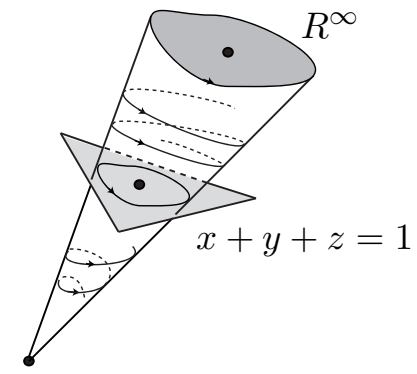

$(0,0,0)$

$(d)$

Figure 1. The global dynamics on the octant $R$ for $a+b=2$ and $0<a<1$.

Theorem 2. The following statements hold for the May-Leonard differential system restricted to $R$ when $a+b=2$ and $(a, b) \neq(1,1)$. All the figures quoted in this theorem corresponds to the case $0<a<1$, for the case $1<a<2$ we must reverse the orientation of all the orbits contained at infinity and at the invariant 2 -dimensional simplex $p(\{x+y+z=1\}) \cap R$.

(a) The phase portrait of the Poincaré compactification of system (1) on the boundaries $p(x=0), p(y=0)$ and $p(z=0)$ of $R$ is topologically equivalent to the one described in Figure 1(a). 
(b) The phase portrait of the Poincaré compactification of system (1) on $R^{\infty}=\partial R \cap\left\{x^{2}+y^{2}+z^{2}=1\right\}$, i.e. the phase portrait at the infinity of $R$, is topologically equivalent to the one described in Figure 1(b). More precisely, the boundary of $R^{\infty}$ is a heteroclinic cycle formed by three equilibrium points coming from the ones located at the end of the three positive half-axes of coordinates, and three orbits connecting these equilibria each one coming from the orbit at the end of every plane of coordinates; in the interior of $R^{\infty}$ we have a center (coming from the end of the invariant bisectriz $x=y=z$ ), its periodic orbits filled completely the interior of $R^{\infty}$.

(c) The plain $x+y+z=1$ is invariant by the flow of system (1). The phase portrait on the 2 -dimensional simplex $R \cap p(\{x+y+z=1\})$ is topologically equivalent to the one described in Figure 1(c). The boundary of this simplex is a heteroclinic cycle formed by the equilibrium points $p((1,0,0)), p((0,1,0))$ and $p((0,0,1))$ located at the vertices of the simplex, and three orbits connecting these equilibria each one on every side of the simplex; in the interior of the simplex we have a center at the equilibrium $p((1 / 3,1 / 3,1 / 3))$, its periodic orbits filled completely the interior of the simplex.

(d) The algebraic surfaces $x y z=h(x+y+z)^{3}$ with $h \in(0,1 / 27]$ are invariant by the flow of system (1), and $R \cap p(x y z=h(x+y+$ $z)^{3}$ ) is homeomorphic to a half-cone $C_{h}$ with vertex at the origin of coordinates and ending at infinity in one of the periodic orbits of the center at infinity drawn in Figure $1(b)$ if $h \in(0,1 / 27)$, and if $h=1 / 27$ than it coincides with $p(x=y=z)$. Every half-cone $C_{h}$ intersect the simplex $R \cap p(\{x+y+z=1\})$ in one of the periodic orbits contained in the simplex. Moreover the orbits on $C_{h}$ below the simplex have their $\alpha$-limit at the equilibrium point $p((0,0,0))$ and their $\omega$-limit at the periodic orbit $p(\{x+y+z=1\}) \cap C_{h}$. The orbits on $C_{h}$ upper the simplex have their $\alpha$-limit in the periodic orbit at the infinity $R^{\infty}$ located at the end of the cone $C_{h}$ and their $\omega$-limit in the periodic orbit $p(\{x+y+z=1\}) \cap C_{h}$. See Figure 1(d). On the invariant line $R \cap p(\{x+y+z=1\})$ the equilibrium $p((1 / 3,1 / 3,1 / 3))$ attracts the two orbits which has at both sides.

The proof of Theorem 2 is given in section 4 .

Basically the new results of Theorem 2 are the description of the flow at infinity of $R$ (statement (b)) and the existence of the invariant cones $C_{h}$ (see statement (d)). The rest of the results can be essentially find in the articles $[1,7,20]$.

The following result describes the trivial dynamics of the May-Leonard differential system when $a=b=1$, and we do not prove it.

Proposition 3. The following statements hold for the May-Leonard differential system (1) when $a=b=1$. 
INTEGRABILITY AND GLOBAL DYNAMICS OF THE MAY-LEONARD MODEL 7

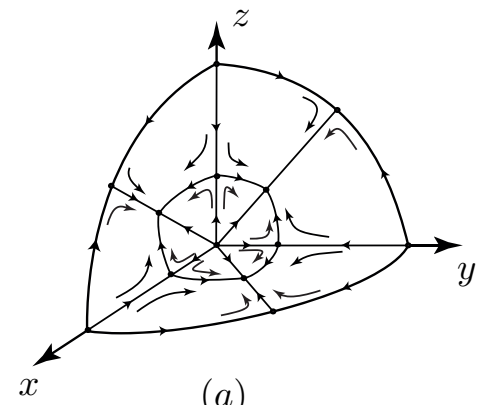

(a)
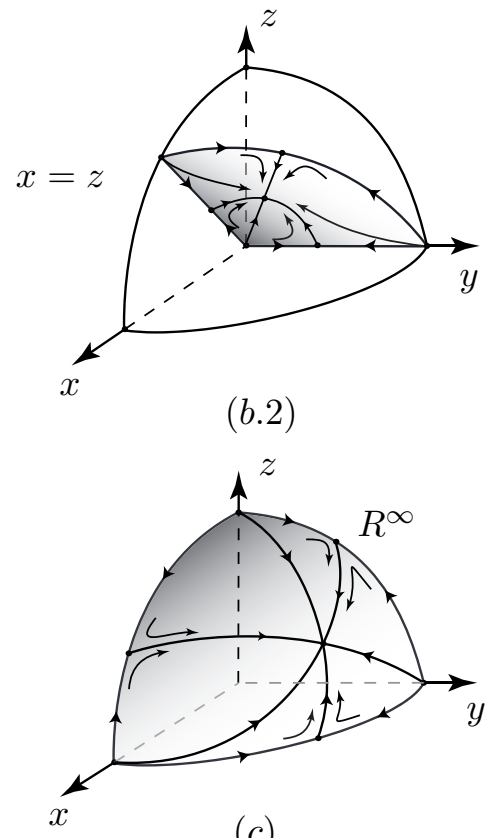

(c)

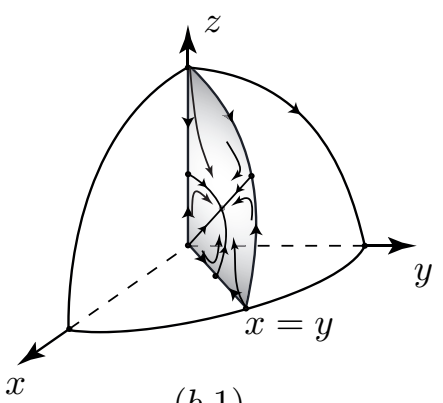

$(b .1)$
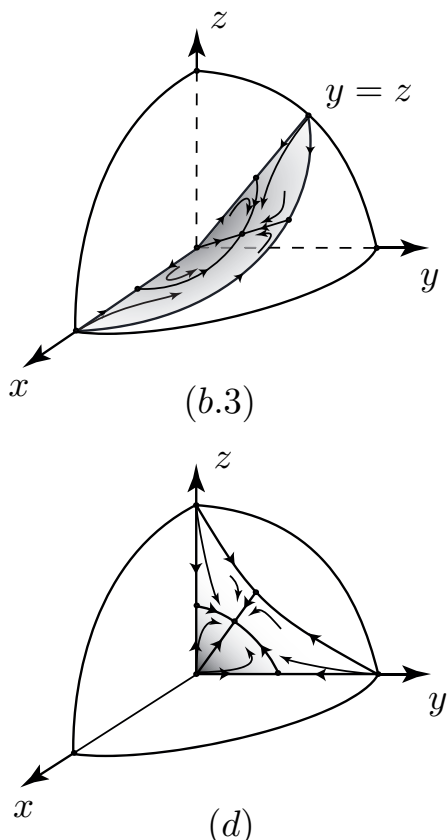

$(d)$

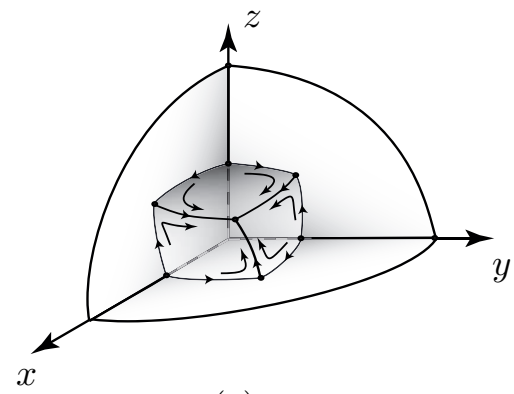

$(e)$

Figure 2. The global dynamics on the octant $R$ for $b=a<1$.

(a) All the straight lines through the origin are invariant. 
(b) Let $\gamma$ be an straight line through the origin of $\mathbb{R}^{3}$. Then the flow on $p(\gamma) \cap R$ has three equilibria, two at its endpoints. The third equilibrium is on the simplex $R \cap p(\{x+y+z=1\})$. This last equilibrium attracts the two orbits which has at both sides.

(c) The infinity $R^{\infty}$ of $R$ and the simplex $R \cap p(\{x+y+z=1\})$ are filled of equilibria.

When we talk about a topological hexagon $S$ (respectively sector) we mean that $S$ is homeomorphic to an hexagon (respectively sector). The flow of the May-Leonard differential system on the boundary and in the interior of $R$ is described in the next theorem when $b=a$.

Theorem 4. The following statements hold for the May-Leonard differential system when $b=a \neq 1$.

(a) The phase portrait of the Poincaré compactification $p(\mathcal{X})$ of system (1) on the boundaries $p(x=0) \cap R, p(y=0) \cap R$ and $p(z=0) \cap R$ of $R$ is topologically equivalent to the one described in Figure $2(a)$ if $0<a<1$, and Figure 3(a) if $a>1$.

(b) The planes $x=y, y=z$ and $z=x$ are invariant by the flow of system (1), and the phase portrait of $p(\mathcal{X})$ on $p(x=y) \cap R$, $p(y=z) \cap R$ and $p(z=x) \cap R$ are topologically equivalent to the ones described in (b.1), (b.2) and (b.3) of Figure 2 if $0<a<1$, and Figure 3 if $a>1$.

(c) The phase portrait of $p(\mathcal{X})$ on $R^{\infty}$, i.e. the phase portrait at the infinity of $R$, is topologically equivalent to the one described in Figure 2(c) if $0<a<1$, and Figure 3(c) if $a>1$.

(d) The algebraic surfaces $y(x-z)=h x(y-z)$ with $h \in \mathbb{R}$ are invariant by the flow of system (1) and they are elliptic cones for $h \neq 0,1$. There are three kinds of topological cones $B \cap p(y(x-z)=h x(y-z))$ in the Poincaré ball. First the ones that restricted to $R \cap p(y(x-z)=$ $h x(y-z))$ contain the image in the Poincaré ball of the half-axes $y$ and $z$, the negative half-axis $x$, and the positive part of the bisectrix $x=y=z$. The other two kinds are obtained from the first kind permuting cyclically the letters $x, y$ and $z$.

The first kind of topological cones $B \cap p(y(x-z)=h x(y-z))$ restricted to $R$ are topological sectors $S_{h}$ with vertex at the origin $p((0,0,0))$, its two sides are the image in the $R$ of the positive halfaxes $y$ and $z$, all the sectors contain the image in $R$ of the positive part of the bisectrix $x=y=z$, and their boundary at infinity. The other two kinds of topological cones also intersect to $R$ in topological sectors which can be described as in the first kind permuting cyclically the letters $x, y$ and $z$.

The flow on one of these sectors of the first kind $S_{h}$ is topologically equivalent to the one described in Figure 2(d) if $0<a<1$, and 
INTEGRABILITY AND GLOBAL DYNAMICS OF THE MAY-LEONARD MODEL 9

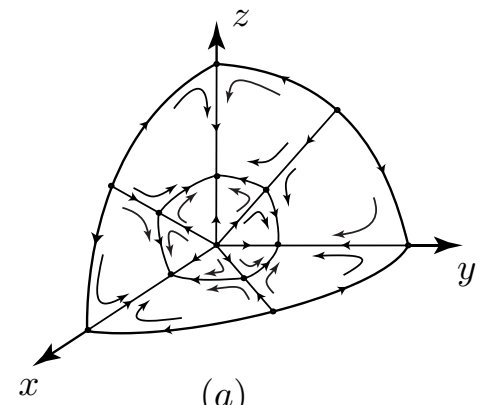

(a)
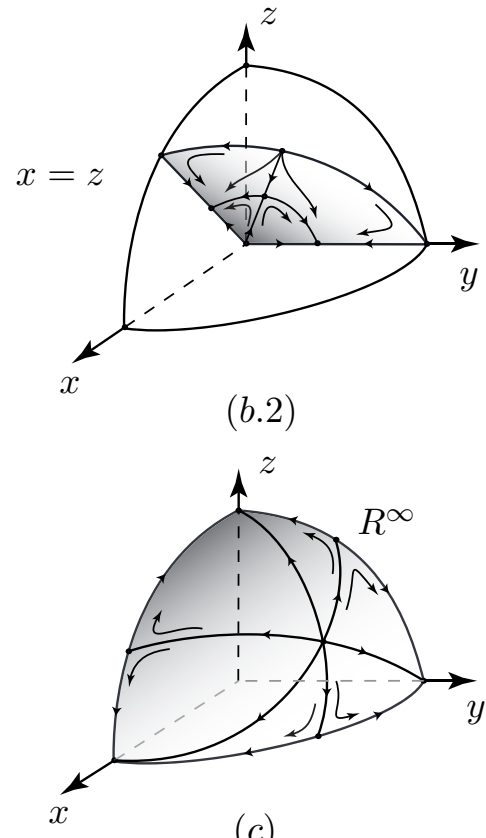

(c)

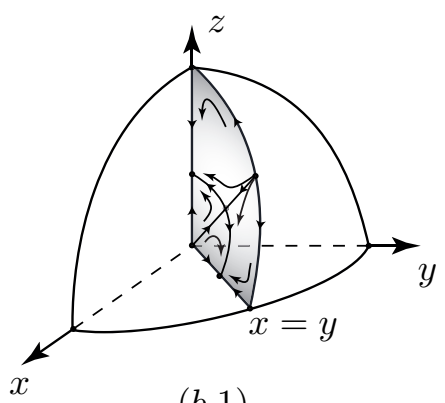

$(b .1)$
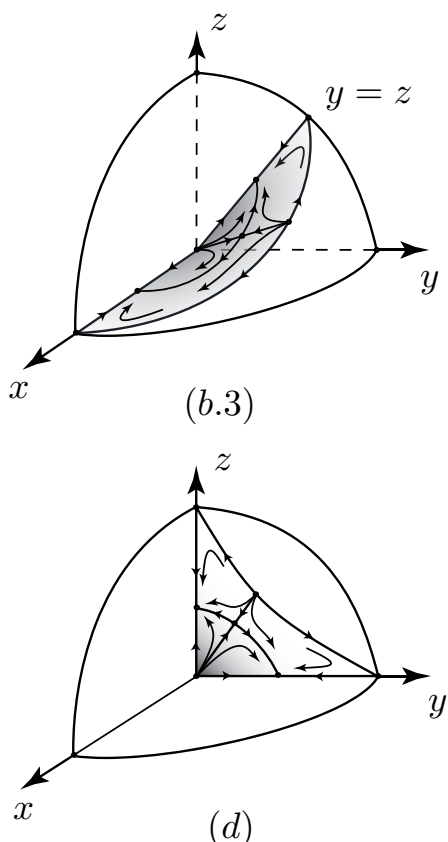

$(d)$

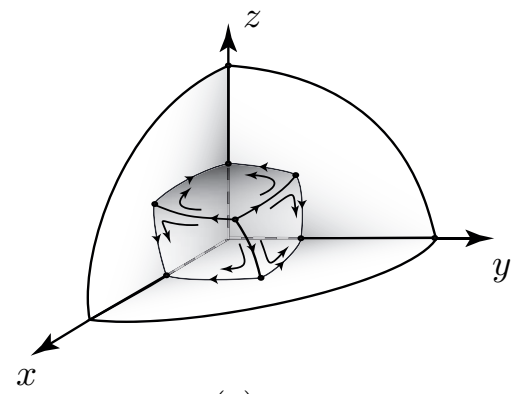

$(e)$

Figure 3 . The global dynamics on the octant $R$ for $b=a>1$.

Figure 3(d) if $a>1$. Similar figures can be drawn for the sectors of the other two kinds. 
(e) If $0<a<1$ then all orbits contained in the interior of $R$ have their $\omega$-limit at $P=p((1 /(1+2 a), 1 /(1+2 a), 1 /(1+2 a)))$.

Assume $a>1$. If

$C=(R \cap p(\{x=y \geq z\})) \cup(R \cap p(\{y=z \geq x\})) \cup(R \cap p(\{z=x \geq y\}))$, then all the orbits contained in the interior of $R \backslash C$ have their $\omega$-limit in one of the following three attractor equilibra $p((1,0,0)), p((0,1,0))$ and $p((0,0,1))$. The three bassins of attraction of these equilibria are separated by the set $C$.

(f) For $0<a<1$ there exists an invariant topological hexagon $S$ of consecutive vertices the equilibria $p((1,0,0)), p((1 /(1+a), 0,1 /(1+$ $a))), p((0,0,1)), p((0,1 /(1+a), 1 /(1+a))), p((0,1,0))$ and $p((1 /(1+$ $a), 1 /(1+a), 0))$, and sides on $p(x=0) \cap R, p(y=0) \cap R$ and $p(z=0) \cap R$. The vertices alternate saddles with repeller nodes being $p((1,0,0))$ a repelling node. In the interior of this topological hexagon there is the equilibrium $P$ which is an attracting node. The flow on $S$ is topologically equivalent to the one described in Figure 2(e).

For $a>1$ there exists an invariant topological hexagon $S$ with the same vertices and sides on the planes of coordinates. The vertices alternate saddles with attracting nodes being $(1,0,0)$ an attracting node. In the interior of this topological hexagon there is the equilibrium $p$ which is a repeller node. The flow on $S$ is topologically equivalent to the one described in Figure 3(e).

The proof of Theorem 4 is given in section 5 .

Statement (f) of Theorem 4 is not relevant for describing the global dynamics of the May-Leonard differential system when $a=b \neq 1$, but it shows that the invariant surface given by the simplex $p(\{x+y+z=1\}) \cap R$ which plays a key role for describing the global dynamics when $a+b=2$, persists now in the invariant surface given by the topological hexagon.

\section{Proof of Theorem 1}

The results used in this section works for differential systems in $\mathbb{R}^{n}$, but here are presented for the May-Leonard differential system (1) defined on the octant $R$ given in (2).

The proof of Theorem 1 needs to recall some classical results on integrability due to Jacobi and Whittaker, see for more details the book of Goriely [5].

Let $J=J(x, y, z)$ be a non-negative $C^{1}$ function non-identically zero on any open subset whose domain of definition is an open and dense subset of $R$. Then $J$ is a Jacobi multiplier of the differential system (1) in $R$ if

$$
\int_{\Omega} J(x, y, z) d x d y d z=\int_{\varphi_{t}(\Omega)} J(x, y, z) d x d y d z,
$$


where $\Omega$ is any open subset of $R$ and $\varphi_{t}$ is the flow defined by the differential system (1) in $R$.

The following result goes back to Jacobi, for a proof see Theorem 2.7 of [5].

Theorem 5 (Jacobi Theorem). Consider the differential system (1) in R, and assume that it admits a Jacobi multiplier $J=J(x, y, z)$ and one first integral. Then the system admits an additional first integral functionally independent with the previous one. That is, the differential system (1) in $R$ is completely integrable.

From (3) the divergence of the May-Leonard vector field is

$$
\operatorname{div}=\frac{\partial P_{1}}{\partial x}+\frac{\partial P_{2}}{\partial y}+\frac{\partial P_{3}}{\partial z}=3-(2+a+b)(x+y+z) .
$$

In general given a function $J$ it is not easy to verify (4). We have the following result of Whittaker [25], which plays a main role for detecting Jacobi multipliers.

Proposition 6. Let $J=J(x, y, z)$ be a non-negative $C^{1}$ function nonidentically zero on any open subset of $R$ whose domain of definition is dense in $R$. Then $J$ is a Jacobi multiplier of the differential system (1) if and only if the divergence of the differential system

$$
\begin{aligned}
& \dot{x}=J(x, y, z) x(1-x-a y-b z), \\
& \dot{y}=J(x, y, z) y(1-b x-y-a z), \\
& \dot{z}=J(x, y, z) z(1-a x-b y-z),
\end{aligned}
$$

is zero.

One of the best tools to look for first integrals is the Darboux theory of integrability. Now we shall introduce the basic notions of this theory which will allow us to compute the Jacobi multipliers. Let $\mathbb{R}[x, y, z]$ be the ring of polynomials in the variables $x, y, z$ with coefficients in $\mathbb{R}$. We say that $f=0$ with $f \in \mathbb{R}[x, y, z]$ is an invariant algebraic surface of the vector field $\mathcal{X}$ if there exists a polynomial $K \in \mathbb{R}[x, y, z]$ such that $\mathcal{X} f=K f$. The polynomial $K=K(x, y, z)$ is called the cofactor of $f$. It is easy to see that since the May-Leonard system (1) is a polynomial differential system of degree 2, every cofactor has degree at most 1 . Of course, an invariant algebraic surface $f=0$ with degree of $f$ equal to one, is an invariant plane.

Since on the points of an invariant algebraic surface $f=0$ the gradient $(\partial f / \partial x, \partial f / \partial y, \partial f / \partial z)$ of the surface is orthogonal to the vector field $\mathcal{X}$ because $\mathcal{X} f=K f$, the vector field $\mathcal{X}$ is tangent to the surface $f=0$. Hence, if an orbit of the vector field $\mathcal{X}$ has a point on the surface $f=0$ the whole orbit is contained in the surface $f=0$. This justifies the name of invariant algebraic surface given to the algebraic surface $f=0$ satisfying $\mathcal{X} f=K f$ for some polynomial $K$, because this surface is invariant under the flow defined by $\mathcal{X}$. 
In the proof of Theorem 1 we will use the following well known result of the Darboux theory of integrability, see for instance [4, Chapter 8], there is it proved for polynomial vector fields in $\mathbb{R}^{2}$, but the same proof works for polynomial vector fields in $\mathbb{R}^{n}$, and in particular for the May-Leonard vector field $\mathcal{X}$.

Theorem 7 (Darboux theory of integrability). Suppose that the polynomial vector field $\mathcal{X}$ defined in $\mathbb{R}^{3}$ admits $p$ invariant algebraic surfaces $f_{i}=0$ with cofactors $K_{i}$ for $i=1, \ldots, p$. If there exist $\lambda_{i} \in \mathbb{R}$ not all zero such that

$$
\sum_{i=1}^{p} \lambda_{i} K_{i}=-\operatorname{div}
$$

then the following function

$$
\left|f_{1}\right|^{\lambda_{1}} \cdots\left|f_{p}\right|^{\lambda_{p}}
$$

is a Jacobi multiplier of the vector field $\mathcal{X}$.

We note that a Jacobi multiplier of a vector field is usually called an integrating factor if the vector field is defined in $\mathbb{R}^{2}$.

Proof of Theorem 1. If $a=b=1$ since $H_{3}$ and $H_{4}$ are two independent first integrals of system (1), it is completely integrable.

Assume that $b=2-a$ and $(a, b) \neq(1,1)$. Then the May-Leonard vector field $\mathcal{X}$ has four invariant planes $f_{i}=0$ with cofactors $K_{i}$, namely

$$
\begin{array}{ll}
f_{1}=x, & K_{1}=1-x-a y-b z ; \\
f_{2}=y, & K_{2}=1-b x-y-a z ; \\
f_{3}=z, & K_{3}=1-a x-b y-z ; \\
f_{4}=x+y+z-1, & K_{4}=-x-y-z
\end{array}
$$

Since $\sum_{i=1}^{4}-K_{i}=-$ div, by Theorem 7 we obtain that

$$
J=J(x, y, z)=\frac{1}{x y z|x+y+z-1|},
$$

is a Jacobi multiplier of $\mathcal{X}$ in $R$ when $b=2-a$ and $(a, b) \neq(1,1)$. This can be checked directly seeing that the divergence of the differential system (5) is zero. Now, since system (1) in $R$ has the first integral $H_{1}$ and a Jacobi multiplier, by Theorem 5 , it is completely integrable in $R$. This proves the theorem when $a+b=2$ and $(a, b) \neq(1,1)$. 
Assume that $b=a$ and $(a, b) \neq(1,1)$. Then the May-Leonard vector field $\mathcal{X}$ has four invariant planes $f_{i}=0$ with cofactors $K_{i}$, namely

$$
\begin{array}{ll}
f_{1}=x, & K_{1}=1-x-a y-b z \\
f_{2}=y, & K_{2}=1-b x-y-a z \\
f_{3}=z, & K_{3}=1-a x-b y-z ; \\
f_{4}=x-z, & K_{4}=1-x-a y-z ; \\
f_{5}=y-z, & K_{5}=1-a x-y-z \\
f_{6}=x-y, & K_{6}=1-x-y-a z
\end{array}
$$

Since

$$
\frac{a+1}{1-a} K_{1}+\frac{a-2}{1-a} K_{2}+\frac{a+1}{1-a} K_{3}+\frac{3}{a-1} K_{4}=-\operatorname{div},
$$

by Theorem 7 we obtain that

$$
J=J(x, y, z)=x^{\frac{a+1}{1-a}} y^{\frac{a-2}{1-a}} z^{\frac{a+1}{1-a}}|x-z|^{\frac{3}{a-1}},
$$

is a Jacobi multiplier of $\mathcal{X}$ in $R$ when $b=a$ and $(a, b) \neq(1,1)$. Again this can be checked directly seeing that the divergence of the differential system 5 is zero. Now, since system (1) in $R$ has the first integral $H_{2}$ and a Jacobi multiplier, by Theorem (5), it is completely integrable in $R$. This proves the theorem when $b=a$ and $(a, b) \neq(1,1)$.

\section{Proof of Theorem 2}

In this section we assume that $b=2-a, 0<a<2$ and $a \neq 1$.

First, we shall study the finite equilibrium points of the May-Leonard system (1) contained in $\mathbb{R}_{+}^{3}$, i.e. we must study the solutions of the system

$$
\begin{aligned}
& x(1-x-a y-(2-a) z)=0, \\
& y(1-(2-a) x-y-a z)=0, \\
& z(1-a x-(2-a) y-z)=0 .
\end{aligned}
$$

They are

$$
p_{0}=(0,0,0), p_{1}=(1,0,0), p_{2}=(0,1,0), p_{3}=(0,0,1), p_{4}=\left(\frac{1}{3}, \frac{1}{3}, \frac{1}{3}\right) .
$$

An equilibrium point $p$ of the May-Leonard differential system is hyperbolic if all the eigenvalues of the Jacobian matrix of the system at $p$ have nonzero real part. The Grobman-Hartman Theorem (see for instance [19]) reduces the study of the local phase portrait at a hyperbolic singular point to study the phase portrait of its linear part. More precisely, the local phase portrait in a sufficiently small neighborhood of a hyperbolic equilibrium point $p$ of a $C^{1}$ differential system is homeomorphic to the phase portrait of the linear part of the differential system at $p$. Using this theorem we obtain the following results.

The eigenvalue at $p_{0}$ is 1 with multiplicity three. So, locally $p_{0}$ is a repeller. Note that the three positive half-axis of coordinates are formed by orbits because the coordinates planes are invariant. Then, the orbits on these three half-axis near $p_{0}$ have $\alpha$-limit $p_{0}$. 
The eigenvalues at $p_{1}$ are $-1, a-1$ and $1-a$. Since the planes of coordinates and the plane $x+y+z=1$ are invariant (see section 3), their intersections are invariant, and consequently formed by orbits.

Assume now that $0<a<1$. Studying the eigenvectors of the Jacobian matrix at $p_{1}$, we obtain that the two orbits at both sides of $p_{1}$ on the $x$-axis have $\omega$-limit $p_{1}$. The open segment with endpoints $p_{1}$ and $p_{2}$ is formed by an orbit with $\omega$-limit $p_{1}$ (and consequently $\alpha$-limit $p_{2}$ ). The open segment with endpoints $p_{1}$ and $p_{3}$ is formed by an orbit whose $\alpha$-limit is $p_{1}$.

If $1<a<2$ then we must reverse the orientation of the orbits living in the two previous segments contained in the plane $x+y+z=1$.

The local behavior at the equilibrium points $p_{2}$ and $p_{3}$ can be studied as we did for $p_{1}$. In this way we obtain that on boundary of $\{x+y+z=1\} \cap \mathbb{R}_{+}^{3}$ we have a heteroclinic cycle formed by the three equilibria $p_{k}$ for $k=1,2,3$, and the three orbits living on the three open segments connecting these three points. The orientation of this cycle is different for $0<a<1$ than for $1<a<2$.

The equilibrium point $p_{4}$ lives in the invariant plane $x+y+z=1$. We want to study the dynamics on this invariant plane. Taking $z=1-x-y$ the May-Leonard differential system restricted to this plane becomes

$$
\begin{aligned}
& \dot{x}=(1-a) x(-1+x+2 y), \\
& \dot{y}=(a-1) y(-1+2 x+y) .
\end{aligned}
$$

Doing a rescaling of the time variable we must study the equilibrium point $(1 / 3,1 / 3)$ of the system

$$
\begin{aligned}
& \dot{x}=-x(-1+x+2 y), \\
& \dot{y}=y(-1+2 x+y) .
\end{aligned}
$$

We translate the equilibrium $(1 / 3,1 / 3)$ at the origin of coordinates doing the change of variables $x=X+1 / 3$ and $y=Y+1 / 3$. In the new variables the system writes

$$
\begin{aligned}
& \dot{X}=-\frac{1}{3}(1+3 X)(X+2 Y), \\
& \dot{Y}=\frac{1}{3}(2 X+Y)(1+3 Y) .
\end{aligned}
$$

We shall write the linear part at the origin of this system in its real Jordan normal form. So, we do the change of variables $X=v$ and $Y=-\sqrt{3} u / 2-$ $v / 2$, and the system becomes

$$
\begin{aligned}
\dot{u} & =-v+\frac{3}{2}\left(v^{2}-u^{2}\right), \\
\dot{v} & =u(1+3 v) .
\end{aligned}
$$

For a proof of the next result see Theorem 8.15 of [4].

Theorem 8 (Kapteyn-Bautin Theorem). A quadratic system that has a center at the origin can be written in the form

$$
\dot{x}=-y-b x^{2}-C x y-d y^{2}, \quad \dot{y}=x+a x^{2}+A x y-a y^{2} .
$$


This system has a center at the origin if and only if at least one of the following conditions holds

(i) $A-2 b=C+2 a=0$,

(ii) $C=a=0$,

(iii) $b+d=0$,

(iv) $C+2 a=A+3 b+5 d=a^{2}+b d+2 d^{2}=0$.

The differential system (8) satisfies condition (iii) of Theorem 8, so it has a center at the origin. Since the Poincaré map around this center is analytic and coincides with the identity, the periodic orbits surrounding this center reaches the previous heteroclinic cycle. The orientation of these periodic orbits coincides with the orientation of the heteroclinic cycle. This completes the proof of statement (c) of Theorem 2.

Proof of statement (b) of Theorem 2. We shall study the dynamics on the infinity $R^{\infty}$ of $R$ using the Poincaré compactification, see for more details the appendix.

Thus our differential system (1) in the local chart $U_{1}$ writes

$$
\begin{aligned}
& \dot{z}_{1}=(a-1) z_{1}\left(1+z_{1}-2 z_{2}\right), \\
& \dot{z}_{2}=(a-1) z_{2}\left(-1+2 z_{1}-z_{2}\right), \\
& \dot{z_{3}}=z_{3}\left(1+a\left(z_{1}-z_{2}\right)+2 z_{2}-z_{3}\right),
\end{aligned}
$$

see for more details equations (14). In $R^{\infty} \cap U_{1}$ the coordinates satisfy $z_{1} \geq 0, z_{2} \geq 0$ and $z_{3}=0$. So the equilibrium points in $R^{\infty} \cap U_{1}$ are $(0,0,0)$ and $(1,1,0)$.

The equilibrium point $(0,0,0)$ is located at the end of the $x$-axis, their eigenvalues are $a-1,1-a$ and 1 with eigenvectors $(1,0,0),(0,1,0)$ and $(0,0,1)$ respectively. Since the planes of coordinates and the sphere at infinity are invariant, their intersection is formed by orbits. So, if $0<a<1$ the open arc of $R^{\infty}$ corresponding at the end of the plane $z=0$ is formed by an orbit having as $\omega$-limit the equilibrium $(0,0,0)$, and the open arc of $R^{\infty}$ corresponding at the end of the plane $y=0$ is formed by an orbit having as $\alpha$-limit the equilibrium $(0,0,0)$. The orbit on the $x$-axis near $(0,0,0)$ has this equilibrium as its $\alpha$-limit. Similar studies can be done for the equilibria located at the origin of the local charts $U_{2}$ and $U_{3}$, i.e. at the end of the $y-$ and $z$-axis respectively. Thus the boundary of $R^{\infty}$ is formed by a heteroclinic cycle formed by three equilibria coming from the ones located at the end of the positive half-axes, and the three orbits living on the three open arcs connecting these three points and contained in the boundary of $R^{\infty}$. The orientation of this heteroclinic cycle is different for $0<a<1$ than for $1<a<2$.

We claim that the local phase portrait at the equilibrium $(1,1,0)$ of the local chart $U_{1}$, and consequently located at the end of the bisectrix $x=$ $y=z$, restricted to the infinity is a center. Moreover, since the bisectrix $x=y=z$ is invariant by the flow and the eigenvalues of the equilibrium 
$(1,1,0)$ are 3 and $\pm \sqrt{3}(a-1) i$, the orbit on the bisectrix near the equilibrium $(1,1,0)$ has this equilibrium as its $\alpha$-limit.

Now we prove the claim. We restrict the differential system (9) to infinity, i.e. to $z_{3}=0$, and we translate the equilibrium $(1,1)$ of this restricted system at the origin doing the change of variables $z_{1}=Z_{1}+1$ and $z_{2}=Z_{2}+1$. Then, in the new coordinates we get the system

$$
\begin{aligned}
& \dot{Z}_{1}=(a-1)\left(1+Z_{1}\right)\left(Z_{1}-2 Z_{2}\right), \\
& \dot{Z}_{2}=(a-1)\left(1+Z_{2}\right)\left(2 Z_{1}-Z_{2}\right) .
\end{aligned}
$$

We remove the constant $a-1$ doing a rescaling of the time. Doing the change of variables $Z_{1}=v$ and $Z_{2}=-\sqrt{3} u / 2+v / 2$ we write the linear part of the previous system in its real Jordan normal, and after dividing it by $\sqrt{3}$ doing again a rescaling of the time we get the system

$$
\begin{aligned}
& \dot{u}=-v+\frac{1}{2}\left(u^{2}-v^{2}\right)+\frac{2}{\sqrt{3}} u v, \\
& \dot{v}=u(1+v) .
\end{aligned}
$$

Since this system satisfies condition (iii) of Theorem 8 , its origin is a center, and using the same arguments than in the proof of statement (c) the image of the periodic orbits of this center in $R^{\infty}$ filled the whole interior of $R^{\infty}$. This completes the proof of the claim, and consequently of statement (b).

Proof of statement (a) of Theorem 2. Putting together the information on the local phase portraits on the five equilibria that there are on the compactified plane $x=0$ intersection with $R$, three finite equilibria and two infinite ones at the endpoints of the positive half-axes $y$ and $z$. Plus the information given by the Poincaré-Bendixson Theorem (see for instance Corollary 1.30 of [4]) we obtain easily the global phase portrait on $p(x=0) \cap R$ described in Figure 1(a).

In a similar way we obtain the global phase portraits on $p(y=0) \cap R$ and $p(z=0) \cap R$ described in Figure 1(a). So this statement is proved.

Proof of statement $(d)$ of Theorem 2. Since $H_{1}=x y z /(x+y+z)^{3}$ is a first integral the algebraic surfaces $S_{h}=\left\{(x, y, z) \in \mathbb{R}_{+}^{3}: x y z=h(x+y+z)^{3}\right\}$ are invariant by the flow. Note that if a point $(x, y, z)$ belongs to $c_{h}$, then the whole half-straight line starting at the origin and passing through this point is contained in $c_{h}$. Take one of these half-straight lines contained in $c_{h}$, it intersects the plane $x+y+z=1$ in a point, the periodic orbit through this point is also contained in $c_{h}$. Therefore, $C_{h}=p\left(c_{h}\right)$ is homeomorphic to a half-cone having its vertex at $p(0,0,0)$, and ending in one of the periodic orbits of the infinity $R^{\infty}$.

It is easy to check that $c_{h}$ is not empty if and only $h \in(0,1 / 27)$, that for $h=1 / 27$ the topological cone $c_{h}$ degenerates in the bisectrix $x=y=z$, and that for $h \in(0,1 / 27)$ the cones $c_{h}$ foliated the interior of $\mathbb{R}_{+}^{3}$. 
Again using the Poincaré-Bendixson Theorem on every invariant topological cone $C_{h}$ we obtain on each of it the dynamics described in statement (d) of Theorem 2. This completes the proof of the statement.

\section{Proof of Theorem 4}

In all this section we assume $b=a \neq 1$ and $a>0$. Then the May-Leonard differential system reduces to

$$
\begin{aligned}
& \dot{x}=x(1-x-a(y+z)), \\
& \dot{y}=y(1-y-a(x+z)), \\
& \dot{z}=z(1-z-a(x+y)) .
\end{aligned}
$$

This system has exactly eight finite singular points, namely

$$
\begin{aligned}
& (0,0,0),(1,0,0),(0,1,0),(0,0,1),\left(\frac{1}{1+2 a}, \frac{1}{1+2 a}, \frac{1}{1+2 a}\right), \\
& \left(\frac{1}{1+a}, \frac{1}{1+a}, 0\right),\left(\frac{1}{1+a}, 0, \frac{1}{1+a}\right),\left(0, \frac{1}{1+a}, \frac{1}{1+a}\right) .
\end{aligned}
$$

The linear part at the equilibrium $(0,0,0)$ has a unique eigenvalue 1 with multiplicity three. Therefore, it is a repelling equilibrium.

The eigenvalues of the linear part at the equilibria $(1,0,0),(0,1,0)$ and $(0,1,0)$ are -1 and $1-a$ with multiplicity two. When $a<1$ these equilibria have an 1-dimensional stable manifold and a 2-dimensional unstable one, and for $a>1$ these equilibria are attractors.

The eigenvalues of the linear part at the equilibrium $(1 /(1+2 a),(1 /(1+$ $2 a),(1 /(1+2 a))$ are -1 and $(a-1) /(1+2 a)$ with multiplicity two. Therefore, this equilibrium is an attractor if $a<1$, and it has an 1-dimensional stable manifold and a 2-dimensional unstable one if $a>1$.

The eigenvalues of the linear part at the equilibria $(1 /(1+a), 1 /(1+a), 0)$, $(1 /(1+a), 0,1 /(1+a)),(0,1 /(1+a), 1 /(1+a))$ are $-1,(a-1) /(1+a)$ and $(1-a) /(1+a)$. Therefore they have a 2 -dimensional stable manifold and an 1-dimensional unstable one.

Now we shall study the infinite equilibrium points of the May-Leonard differential system (10), and we start to prove the different statements of Theorem 4.

Proof of statement $(c)$ of Theorem 4. In the local chart $U_{1}$ the differential system (10) becomes

$$
\begin{aligned}
& \dot{z_{1}}=(a-1) z_{1}\left(z_{1}-1\right), \\
& \dot{z_{2}}=(a-1) z_{2}\left(z_{2}-1\right), \\
& \dot{z_{3}}=z_{3}\left(1+a\left(z_{1}+z_{2}\right)-z_{3}\right) .
\end{aligned}
$$

Recall that the infinity $R^{\infty}$ in any local chart corresponds to the invariant plane $z_{3}=0$.

System (11) has four equilibria at infinity: $(0,0,0),(1,0,0),(0,1,0)$ and $(1,1,0)$. The linear part at the equilibrium $(0,0,0)$ has the eigenvalue $1-a$ 
with multiplicity 2 at infinity and eigenvalue 1 in its finite direction. Therefore, on the infinity $(0,0,0)$ is an unstable (respectively stable) node if $a<1$ (respectively if $a>1$ ).

The eigenvalues of the linear part at the equilibrium $(1,0,0)$ are $a-1$ and $1-a$ at infinity and eigenvalue $a+1$ in its finite direction. Therefore, on the infinity $(1,0,0)$ is a saddle such that its stable (respectively unstable) separatrix is contained in the $z_{1}$-axis when $a<1$ (respectively when $a>1$ ).

The linear part at the equilibrium $(0,1,0)$ has eigenvalues $1-a$ and $a-1$ at infinity and eigenvalue $a+1$ in its finite direction. Therefore, on the infinity it is a saddle whose stable (respectively unstable) separatrix is contained in the $z_{2}$-axis if $a<1$ (respectively when $a>1$ ).

The eigenvalues of the linear part at the equilibrium $(1,1,0)$ are $a-1$ with multiplicity 2 at infinity, and $2 a+1$ in its finite direction. Therefore, on the infinity it is a stable (respectively unstable) node if $a<1$ (respectively when $a>1)$.

Note that the previous four equilibria are unstable in their finite direction.

The local chart $U_{1}$ does not covers the end part of the plane $x=0$ at the infinity of $\mathbb{R}_{+}^{3}$. So we shall consider the expression of the May-Leonard differential system in the local chart $U_{2}$. Both charts together covers all the infinity of $\mathbb{R}_{+}^{3}$ except the equilibrium at the endpoint of the invariant positive half-axis $z$.

In the local chart $U_{2}$ restricted to the infinity of $\mathbb{R}_{+}^{3}$ the system (10) writes

$$
\begin{aligned}
& \dot{z_{1}}=(a-1) z_{1}\left(z_{1}-1\right), \\
& \dot{z_{2}}=(a-1) z_{2}\left(z_{2}-1\right), \\
& \dot{z_{3}}=z_{3}\left(1+a\left(z_{1}+z_{2}\right)-z_{3}\right) .
\end{aligned}
$$

We are interested only in the equilibria which are on $z_{1}=0$ and $z_{3}=0$. There are two of such equilibria $(0,0,0)$ and $(0,1,0)$.

The eigenvalues of the linear part at the equilibrium $(0,0,0)$ are $1-a$ with multiplicity 2 at infinity, and 1 in its finite direction. Therefore, on the infinity it is an unstable (respectively stable) node if $a<1$ (respectively when $a>1$ ).

The linear part at the equilibrium $(0,1,0)$ has the eigenvalues $1-a$ and $a-1$ at infinity, and $a+1$ in its finite direction. Therefore, on the infinity this equilibrium is a saddle having its stable (respectively unstable) separatrix contained in the $z_{2}$-axis if $a<1$ (respectively when $a>1$ ).

Note again that the previous two equilibria are unstable in their finite direction.

Now we only need to study the equilibrium at the endpoint of positive $z$-half-axis, i.e. the equilibrium at the origin of the local chart $U_{3}$. In this 
chart system (10) becomes

$$
\begin{aligned}
& \dot{z_{1}}=(a-1) z_{1}\left(z_{1}-1\right), \\
& \dot{z_{2}}=(a-1) z_{2}\left(z_{2}-1\right), \\
& \dot{z_{3}}=z_{3}\left(1+a\left(z_{1}+z_{2}\right)-z_{3}\right) .
\end{aligned}
$$

The linear part at the equilibrium $(0,0,0)$ has eigenvalues $1-a$ with multiplicity 2 at infinity, and 1 in its finite direction. Therefore, at infinity if $a<1$ (respectively $a>1$ ) then this equilibrium is an unstable (respectively stable) node.

In short taking into account the local behavior at all the infinite equilibria, and the fact that the boundaries of the invariant planes at the infinity $\mathbb{R}_{+}^{3}$ are invariant, it follows the phase portrait at the infinity $R^{\infty}$ described in the Figure 2(c) and Figure 3(c). So the proof of this statement is complete.

Proofs of statements $(a)$ and $(b)$ of Theorem 4. Putting together the information on the local phase portraits of all finite and infinite equilibria living on invariant surfaces $p(x=0) \cap R, p(y=0) \cap R, p(z=0) \cap R, p(x=y) \cap R$, $p(y=z) \cap R$ and $p(z=x) \cap R$ (already obtained in this section), plus the information given by the Poincaré-Bendixson Theorem we obtain easily the global phase portrait on the six invariant mentioned surfaces described in the statements (a) and (b) of Theorem 4.

Proof of statement $(d)$ of Theorem 4. We only prove statement (d) for the sectors of first kind, the proof for the other sectors is completely similar.

Since $H_{2}=y(x-z) /(y(y-z))$ is a first integral when $b=a \neq 1$ (the case that now we are studying), the algebraic surfaces $y(x-z)=h x(y-z)$ with $h \in \mathbb{R}$ are invariant by the flow of system (1). Using the classification of quadrics it easy to check that these quadratic algebraic surfaces are elliptic cones if $h \neq 0,1$.

It is immediate to check that every one of these cones contains the three axes of coordinates plus the bisectrix $x=y=z$. More precisely, the halfcone which intersects the octant $\mathbb{R}_{+}^{3}$ contains the positive half-axes $y$ and $z$, the negative half-axis $x$, and the positive part of the bisectrix $x=y=z$. These cones restricted to $\mathbb{R}_{+}^{3}$ are homeomorphic to a sector $s_{h}$ with vertex at the origin of coordinates, its two sides are the positive half-axes $y$ and $z$, all the sectors contain the positive part of the bisectrix $x=y=z$, and their boundary at infinity. So the finite equilibria $(0,0,0),(0,1,0),(0,0,1)$ and $(1 /(1+2 a), 1 /(1+2 a), 1 /(1+2 a))$ are contained in this sector, together with the three infinite equilibria at the end of the $y$ and $z$ axes, and at the end of the positive part of the bisectrix $x=y=z$. The sector $s_{h}$ is invariant by the flow of the May-Leonard differential system, and we know the local phase portraits of the mentioned equilibria restricted to $s_{h}$, then using the Poincaré-Bendixson Theorem it follows that the flow on one of the sectors $S_{h}=\overline{p\left(s_{h}\right)}$ is topologically equivalent to the one described in Figure 2(d) if $0<a<1$, and Figure 3(d) if $a>1$. Hence, statement (d) is proved. 
Proof of statement (e) of Theorem 4. The three kind of sectors $s_{h}$ of statement (d) together with the invariant planes $x=y, y=z$ and $z=x$ restricted to the octant $\mathbb{R}_{+}^{3}$ foliated it.

If $a<1$ on each of the open leaves of the foliation the equilibrium $(1 /(1+$ $2 a), 1 /(1+2 a), 1 /(1+2 a))$ is an attractor, consequently it is a global attractor in the interior of $\mathbb{R}_{+}^{3}$.

If $a>1$ on each of the open leaves of the foliation two of the three equilibria $(1,0,0),(0,1,0)$ and $(0,0,1)$ attract all the periodic orbits in the interior of $\mathbb{R}_{+}^{3}$, with the exception of the orbits contained in the invariant set

$$
c=\left(\mathbb{R}_{+}^{3} \cap\{x=y \geq z\}\right) \cup\left(\mathbb{R}_{+}^{3} \cap\{y=z \geq x\}\right) \cup\left(\mathbb{R}_{+}^{3} \cap\{z=x \geq y\}\right) .
$$

Therefore, all the orbits contained in the interior of $\mathbb{R}_{+}^{3} \backslash b$ have their $\omega$-limit in one of the following three attractors $(1,0,0),(0,1,0)$ and $(0,0,1)$. The three bassins of attraction of these nodes are separated by the set $b$. The set $C$ of the statement (e) of Theorem 4 is $C=\overline{p(c)}$.

Proof of statement $(f)$ of Theorem 4. As before the three kind of sectors $s_{h}$ of statement (d) together with the invariant planes $x=y, y=z$ and $z=x$ restricted to the octant $\mathbb{R}_{+}^{3}$ foliated it.

Suppose $a<1$. For the sectors $S_{h}=\overline{p\left(s_{h}\right)}$ having the flow described in Figure 2(d), we consider in $s_{h}$ the two separatrices which go to the equilibrium $(1 /(1+2 a), 1 /(1+2 a), 1 /(1+2 a))$ starting at the equilibria $(0,1,0)$ and $(0,0,1)$ moving $h$ continuously these two separatrices define a surface passing through the equilibrium $(1 /(1+2 a), 1 /(1+2 a), 1 /(1+2 a))$. We also have other two surfaces passing through the equilibrium $(1 /(1+2 a), 1 /(1+$ $2 a), 1 /(1+2 a))$ coming from the other two kind of sectors. Pasting together the image of these three surfaces in $R$ we obtain the topological hexagon $S$ defined in statement (f) of Theorem 4.

In a similar way we obtain the corresponding topological hexagon $S$ for $a>1$.

The APpendix: Poincaré COMPACtifichtion of $\mathbb{R}^{3}$

In $\mathbb{R}^{3}$ we consider the polynomial differential system

$$
\dot{x}=P^{1}(x, y, z), \quad \dot{y}=P^{2}(x, y, z), \quad \dot{z}=P^{3}(x, y, z),
$$

or equivalently its associated polynomial vector field $X=\left(P^{1}, P^{2}, P^{3}\right)$. The degree $n$ of $X$ is defined as $n=\max \left\{\operatorname{deg}\left(P^{i}\right): i=1,2,3\right\}$.

Let $\mathbb{S}^{3}=\left\{y=\left(y_{1}, y_{2}, y_{3}, y_{4}\right) \in \mathbb{R}^{4}:\|y\|=1\right\}$ be the unit sphere in $\mathbb{R}^{4}$, and

$$
\mathbb{S}_{+}=\left\{y \in \mathbb{S}^{3}: y_{4}>0\right\} \quad \text { and } \quad \mathbb{S}_{-}=\left\{y \in \mathbb{S}^{3}: y_{4}<0\right\}
$$

be the northern and southern hemispheres, respectively. The tangent space to $\mathbb{S}^{3}$ at the point $y$ is denoted by $T_{y} \mathbb{S}^{3}$. Then, the tangent hyperplane

$$
T_{(0,0,0,1)} \mathbb{S}^{3}=\left\{(x, y, z, 1) \in \mathbb{R}^{4}:(x, y, z) \in \mathbb{R}^{3}\right\}
$$


is identified with $\mathbb{R}^{3}$.

We consider the central projections

$$
f_{+}: \mathbb{R}^{3}=T_{(0,0,0,1)} \mathbb{S}^{3} \rightarrow \mathbb{S}_{+} \quad \text { and } \quad f_{-}: \mathbb{R}^{3}=T_{(0,0,0,1)} \mathbb{S}^{3} \rightarrow \mathbb{S}_{-},
$$

defined by

$$
f_{+}(\mathbf{x})=\frac{1}{\Delta x}(x, y, z, 1) \quad \text { and } \quad f_{-}(\mathbf{x})=-\frac{1}{\Delta x}(x, y, z, 1),
$$

where $\Delta x=\left(1+x^{2}+y^{2}+z^{2}\right)^{1 / 2}$. Through these central projections, $\mathbb{R}^{3}$ can be identified with the northern and the southern hemispheres, respectively. The equator of $\mathbb{S}^{3}$ is $\mathbb{S}^{2}=\left\{y \in \mathbb{S}^{3}: y_{4}=0\right\}$. Clearly, $\mathbb{S}^{2}$ can be identified with the infinity of $\mathbb{R}^{3}$.

The maps $f_{+}$and $f_{-}$define two copies of $X$, one $D f_{+} \circ X$ in the northern hemisphere and the other $D f_{-} \circ X$ in the southern one. Denote by $\bar{X}$ the vector field on $\mathbb{S}^{3} \backslash \mathbb{S}^{2}=\mathbb{S}_{+} \cup \mathbb{S}_{-}$which restricted to $\mathbb{S}_{+}$coincides with $D f_{+} \circ X$ and restricted to $\mathbb{S}_{-}$coincides with $D f_{-} \circ X$.

In what follows we shall work with the orthogonal projection of the closed northern hemisphere to $y_{4}=0$. Note that this projection is a closed ball $B$ of radius one, whose interior is diffeomorphic to $\mathbb{R}^{3}$ and whose boundary $\mathbb{S}^{2}$ corresponds to the infinity of $\mathbb{R}^{3}$. We shall extend analytically the polynomial vector field $\bar{X}$ to the boundary, in such a way that the flow on the boundary is invariant. This new vector field $p(X)$ on $B$ will be called the Poincaré compactification of $X$, and $B$ will be called the Poincaré ball. Poincaré introduced this compactification for polynomial vector fields in $\mathbb{R}^{2}$, and its extension to $\mathbb{R}^{m}$ can be found in [3].

As $\mathbb{S}^{3}$ is a differentiable manifold, to compute the expression for $p(X)$ we can consider the eight local charts $\left(U_{i}, F_{i}\right),\left(V_{i}, G_{i}\right)$ where $U_{i}=\left\{y \in \mathbb{S}^{3}\right.$ : $\left.y_{i}>0\right\}$, and $V_{i}=\left\{y \in \mathbb{S}^{3}: y_{i}<0\right\}$ for $i=1,2,3,4$; the diffeomorphisms $F_{i}: U_{i} \rightarrow \mathbb{R}^{3}$ and $G_{i}: V_{i} \rightarrow \mathbb{R}^{3}$ for $i=1,2,3,4$ are the inverses of the central projections from the origin to the tangent planes at the points $( \pm 1,0,0,0)$, $(0, \pm 1,0,0),(0,0, \pm 1,0)$ and $(0,0,0, \pm 1)$, respectively. Thus the analytical field $p(X)$ in the local chart $U_{1}$ becomes

$$
\frac{z_{3}^{n}}{(\Delta z)^{n-1}}\left(-z_{1} P^{1}+P^{2},-z_{2} P^{1}+P^{3},-z_{3} P^{1}\right),
$$

where $P^{i}=P^{i}\left(1 / z_{3}, z_{1} / z_{3}, z_{2} / z_{3}\right)$. The expressions of $p(X)$ in $U_{2}$ and $U_{3}$. These are

$$
\frac{z_{3}^{n}}{(\Delta z)^{n-1}}\left(-z_{1} P^{2}+P^{1},-z_{2} P^{2}+P^{3},-z_{3} P^{2}\right)
$$

where $P^{i}=P^{i}\left(z_{1} / z_{3}, 1 / z_{3}, z_{2} / z_{3}\right)$ in $U_{2}$, and

$$
\frac{z_{3}^{n}}{(\Delta z)^{n-1}}\left(-z_{1} P^{3}+P^{1},-z_{2} P^{3}+P^{2},-z_{3} P^{3}\right),
$$


where $P^{i}=P^{i}\left(z_{1} / z_{3}, z_{2} / z_{3}, 1 / z_{3}\right)$ in $U_{3}$. The expression for $p(X)$ in $U_{4}$ is $z_{3}^{n+1}\left(P^{1}, P^{2}, P^{3}\right)$ where the component $P^{i}=P^{i}\left(z_{1}, z_{2}, z_{3}\right)$. See the details the Poincaré compactification in $\mathbb{R}^{n}$ in [3], or for an easy deduction in $\mathbb{R}^{2}$ the chapter 5 of [4].

The expression for $p(X)$ in the local chart $V_{i}$ is the same as in $U_{i}$ multiplied by $(-1)^{n-1}$.

When we shall work with the expression of the compactified vector field $p(X)$ in the local charts we omit the factor $1 /(\Delta z)^{n-1}$. We can do that through a rescaling of the time.

We remark that all the points on the sphere at infinity in the coordinates of any local chart have $z_{3}=0$.

The ortogonal projection of the closed north hemisphere of $\mathbb{S}^{3}$ on the hyperplane $y_{4}=0$ is a closed ball $B$ of radius 1 centered at the origin of coordinates, whose interior is diffeomorphic to $\mathbb{R}^{3}=T_{(0,0,0,1)} \mathbb{S}^{3}$ and whose boundary $\mathbb{S}^{2}$ is the infinity of $\mathbb{R}^{3}$. This closed ball $B$ is the Poincaré ball.

\section{ACKNOWLEDGEMENTS}

We thank to the referee his comments and suggestions which allow us to improve the presentation of this article.

The authors are partially supported by the program "Consolidación de Grupos Emergentes" by CONACYT. The third author has been partially supported by the grants MICINN/FEDER MCYT/FEDER MTM2008-03437, AGAUR 2005SGR-00550 and ICREA Academia.

\section{REFERENCES}

[1] A. Battauz and F. Zanolin, Coexistence states for periodic competitive Kolmogorov system, J. Math. Anal. Appl. 219 (1998), 179-199.

[2] F. CAO AND J. JiAnG, The Classification on the global phase portraits of twodimensional Lotka-Volterra system, J. Dyn. Diff. Equat. 20 (2008), 797-830.

[3] A. Cima And J. Llibre, Bounded polynomial vector fields, Trans. Amer. Math. Soc. 318 (1990), 557-579.

[4] F. Dumortier, J. Llibre And J.C. Artés, Qualitative theory of planar differential systems, UniversiText, Springer-Verlag, New York, 2006.

[5] A. Goriely, Integrability and nonintegrability of dynamical systems, Advanced Series in Nonlinear Dynamics 19, World Scientific Publishing Co., Inc., River Edge, NJ, 2001.

[6] M.W. HIRSCH, Systems of differential equations that are competitive or cooperative: III. Competing species, Nonlinearity 1 (1988), 51-71.

[7] J. Hofbauer, On the occurrence of limit cycles in the Volterra-Lotka equation, Nonlinear Anal. Theory Methods Appl. 5 (1981), 1003-1007.

[8] J. Hofbauer and K. Sigmund, Evolutionary Games and Population Dynamics, Cambridge, Cambridge University Press, 1988.

[9] Z. Hou, Permanence and extinction in competitive Lotka-Volterra systems with delays, Nonlinear Anal. Real World Appl. 12 (2011), 2130-2141.

[10] Z. Hou, Oscillations and limit cycles in Lotka-Volterra systems with delays, Nonlinear Anal. 75 (2012), 358-370. 
[11] L.-C. Hung, Traveling wave solutions of competitive-cooperative Lotka-Volterra systems of three species, Nonlinear Anal. Real World Appl. 12 (2011), 3691-3700.

[12] A. Kolmogorov, Sulla teoria di Volterra della lotta per l'esistenza, Giornale dell' Istituto Italiano degli Attuari 7 (1936), 74-80.

[13] P.G.L. Leach And J. MiRITZIS, Analytic Behaviour of competition among three species, J. Nonlinear Math. Phys. 13 (2006), 535-548.

[14] J. Llibre And C. Valls, Polynomial, rational and analytic first integrals for a family of 3-dimensional Lotka-Volterra systems, Z. Angew. Math. Phys 62 (2011), 761-777.

[15] A.J. LотKA, Analytical note on certain rhythmic relations in organic systems, Proc. Natl. Acad. Sci. U.S. 6 (1920), 410-415.

[16] V. Marinca And N. Herisanu, Optimal parametric iteration method for solving multispecies Lotka-Volterra equations, Discrete Dynamics in Nature and Society 2012, art. no 842121.

[17] R.M. MAY AND W.J. LEONARD, Nonlinear aspects of competition between three species, SIAM J. of Appl. Math. 29 (1975), 243-253.

[18] K. Orihashi And Y. AizaWA, Global aspects of turbulence induced by heteroclinic cycles in competitive diffusion Lotka-Volterra equation, Physica D 240 (2011), 18531862.

[19] J. Palis AND W. DE Melo, Geometric theory of dynamical systems: an introduction, Springer-Verlag, 1982.

[20] P. Schuster, K. Sigmund And R. Wolf, On $\omega$-limits for competition between three species, SIAM J. Appl. Math. 37 (1979), 49-54.

[21] A. Tineo, May Leonard systems, Nonlinear Anal.: Real World Appl. 9 (2008), 16121618.

[22] R.M. Tudorana And A. Gîrban, On a Hamiltonian version of a three-dimensional Lotka-Volterra system, Nonlinear Anal.: Real World Appl. 13 (2012), 2304-2312.

[23] O. Vasilyeva AND F. Lutscher, Competition of three species in an advective environment, Nonlinear Anal.: Real World Appl. 13 (2012), 1730-1748.

[24] V. Volterra, Lecons sur la Théorie Mathématique de la Lutte pour la vie, GauthierVillars, Paris, 1931.

[25] E.T. Whittaker, A treatise on the analytic dynamics of particles and rigid bodies, Dover, New York, 1944.

[26] M.L. ZeEman, Hopf bifurcations in competitive three dimensional Lotka-Volterra systems, Dynam. Stab. Sys. 8 (1993), 189-217.

1 División Académica de Ciencias Básicas, UJAT, Km 1 Carretera CunduacÁn-Jalpa de MÉndez, C.P. 86690 Cunduacán Tabasco, MÉxico

E-mail address: gble@ujat.mx, vicas@ujat.mx, xangari82@yahoo.com.mx

2 Departament de Matemátiques, Universitat Autónoma de Barcelona, 08193

Bellaterra, Barcelona, Catalonia, Spain

E-mail address: jllibre@mat.uab.cat 